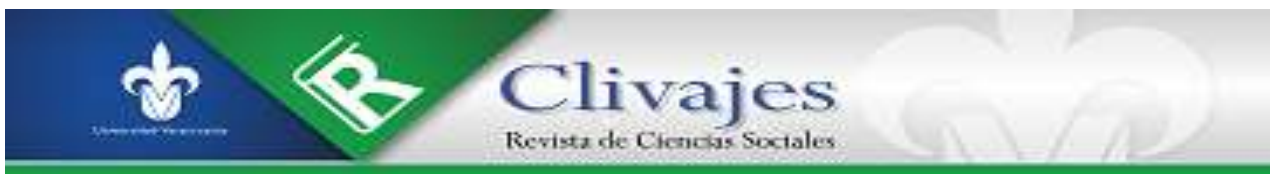

Arturo Narváez Aguilera

POLÍTICAS Y MARCOS LEGALES SOBRE ADOLESCENCIAS Y JUVENTUDES EN MÉXICO: UNA MIRADA SOCIOLÓGICA

Clivajes. Revista de Ciencias Sociales. Año VI, número 12, julio-diciembre 2019, pp. 43-61. http://clivajes.uv.mx/index.php/Clivajes/editor/proofGalley/2582/4457 Instituto de Investigaciones Histórico-Sociales, Universidad Veracruzana

Recibido: 03/09/2019

Aceptado: 10/09/2019

Dictaminado: 15/11/2019

Publicado: 20/12/2019 


\title{
POLÍTICAS Y MARCOS LEGALES SOBRE ADOLESCENCIAS Y JUVENTUDES EN MÉXICO: UNA MIRADA SOCIOLÓGICA
}

\author{
Arturo Narváez Aguilera*
}

\begin{abstract}
Resumen
El artículo sitúa la discusión en la legislación y las políticas hacia los adolescentes y jóvenes en México, con énfasis en la participación política y social. Se analizan aquí las tensiones entre las formas de mediación estatal, condensadas en el paradigma liberal y universalista de los derechos humanos y la pluralidad de experiencias juveniles en contextos multiculturales. En el primer enclave analítico, se problematiza el enfoque de sujetos de derechos a la luz de instrumentos internacionales en la materia, y, enseguida, se abordan los mecanismos estatales de participación de los jóvenes y su convergencia con la trama neoliberal.
\end{abstract}

Palabras clave: Derechos, Juventud, Adolescencia, Subjetividad, Socialización, políticas, Legislación

\section{INTRODUCCIÓN}

Este trabajo aborda, como enclave temático, las políticas y marcos legales sobre las adolescencias y juventudes en México, con una observación situada en la participación social y política en su sentido más amplio, desde una perspectiva analítica sociológica. En el marco del proyecto intitulado "La inclusión social y la ciudadanía de las y los jóvenes en entornos de violencia, vulnerabilidad y exclusión en Veracruz”, es pertinente orientar la discusión en torno a las tensiones, los límites que impone el dispositivo institucional de participación juvenil y las experiencias de organización y protagonismo de los jóvenes en las sociedades modernas latinoamericanas.

Se asume que las subjetividades juveniles trascienden las fronteras de la noción de juventud, en tanto división arbitraria y clasificatoria por edad, propia de la tradición normativa y legal. En consonancia con la perspectiva del sociólogo francés Pierre Bourdieu (1990), pensar la categoría de juventud es ampliar los horizontes analíticos al objeto de lucha en las relaciones intergeneracionales, que se traduce en cómo se condensa la socialización en un movimiento dialéctico, a modo de reproducción del orden y su desbordamiento en estrategias de emancipación. Siguiendo este argumento, la

\footnotetext{
* Sociologo, maestro en Ciencias Sociales (IIH-S, UV) y doctorante en Sociología (Instituto de Ciencias Sociales y Humanidades “Alfonso Vélez Pliego”, BUAP). Es profesor-investigador en la Facultad de Sociología de la UV y participante del proyecto intitulado "La inclusión social y la ciudadanía de las y los jóvenes en entornos de violencia, vulnerabilidad y exclusión en Veracruz".
} 
juventud es una construcción social en disputa; ello implica el reconocimiento de la pluralidad de juventudes y trayectorias que se configuran en multiplicidad de experiencias, las cuales expresan la interiorización de un repertorio diferenciado de disposiciones y hábitos en universos sociales y escenarios múltiples (Lahire, 2004).

El estudio de las experiencias juveniles - como sistema de acción-constituye una coordenada analítica para la comprensión de la complejidad de las relaciones sociales y las representaciones en el campo de lo juvenil, constituidas por las dimensiones de los mecanismos objetivos y subjetivos del mundo social de los jóvenes (Dubet, 2007). A partir del análisis de sus trayectorias, se cuenta con una herramienta conceptual y metodológica, orientada a la focalización de inflexiones/itinerarios de cambio y agenciamiento en los cursos de la biografía juvenil (Ibarrola, 2004, Guerra, 2009). Su aporte, permite develar variaciones y rupturas en el conjunto de toma decisiones de los jóvenes que experimentan acumulación de desigualdades, fragmentación social y encadenamientos de violencias (Misse, 2010, Auyero y Berti, 2013, Saraví, 2015).

Desde nuestra perspectiva, las experiencias juveniles de participación social y política en entornos de violencia, vulnerabilidad y exclusión social transitan por un complejo entramado de formas familiares, barriales y comunitarias que expresan una micropolítica, a escala molecular, en las estructuras de socialización. En este sentido, es pertinente analizar la mediación estatal de la participación, a partir de un corpus de políticas y marcos legales de carácter internacional, nacional y estatal. Más allá de un esfuerzo descriptivo, discutimos las posibilidades y limitaciones del despliegue de postulados, mecanismos y acciones institucionalizadas para la organización e inclusión política de las juventudes veracruzanas.

\section{ADOLESCENTES Y JÓVENES COMO SUJETOS DE DERECHOS}

El tránsito de la noción de objetos de tutela a sujetos de derechos es resultado de un proceso social y político, pleno de luchas y tensiones por el reconocimiento de los jóvenes y adolescentes como titulares de sus derechos, establecidos en la Convención sobre los Derechos del Niño, de la Organizaciones de las Naciones Unidas (CDN,1989), y la Convención Iberoamericana de los Derechos de los Jóvenes, de la Organización Iberoamericana de la Juventud (CDI, 2005). ${ }^{1}$

\footnotetext{
${ }^{1}$ La Convención internacional sobre los Derechos del Niño (CDN), jurídicamente vinculante, firmada por el Estado mexicano en 1989 y ratificada por el Senado en 1990, está precedida por la Declaración de Ginebra sobre los Derechos del Niño (1924) y la Asamblea General de la Liga de las Naciones (1920); se funda en la iniciativa de Eglantyne Jebb, presidenta de Save the Children Fund, la Declaración de los Derechos del Niño, aprobada por la Asamblea General de las Naciones Unidas (ONU) en 1959 y
} 
Es importante precisar que los instrumentos jurídicos de los derechos de las juventudes y las infancias tienen su base ideológica en el pensamiento occidental y eurocéntrico, propio de la Revolución francesa y la Declaración de los Derechos Humanos y Ciudadanos (1789). Al respecto, el sociólogo Manfred Liebel (2006) puntualiza:

la forma en la que hoy se establecen los Derechos del Niño y la manera en la que se los define en legislaciones nacionales y convenciones internacionales, se basan fundamentalmente en el pensamiento de la época de la Ilustración Europea y en el surgimiento de las sociedades burguesas y de los Estados Nacionales en Europa, desde donde se han ido extendiendo por casi la totalidad del mundo (p. 9).

El régimen universal de los derechos humanos en la modernidad occidental se ha sustentado en la visión hegemónica liberal de la persona-individual y los derechos civiles y políticos, que han descansado en una diversidad de instituciones sociales, políticas y jurídicas (Pilotti, 2001). Desde esta noción, los derechos humanos han sido parte de un proceso de fragmentación social y adecuación a las particularidades de lo humano como etapas y desarrollo de la vida del individuo, que ha derivado en una forma de clasificación de marcos jurídicos de derechos por condición de edad: niñez, adolescencia y juventud. ${ }^{2}$

Trascender esta división arbitraria implica analizar los derechos infantiles y juveniles como una construcción sociohistórica en las sociedades modernas e industriales, que responde a los cambios en la posición social de la niñez y los jóvenes con respecto al

el Año Internacional del Niño (1979). Para Liebel (2006), la CDN es resultado de un conjunto de movimientos sociales por los derechos de la infancia; destacan la "Educación Libre para los niños", movimiento pionero en Moscú con la Revolución rusa, que se concreta en la Declaración de Moscú sobre los Derechos del Niño y la Niña, 1918; los movimientos de los años setenta y ochenta: “Movimiento por la liberación de los niños" (Children's Liberations Movement_CLM) en Estados Unidos, además de movimientos latinoamericanos: Movimiento de Adolescentes y Niños Trabajadores Hijos de Obreros Cristianos (MANTHOC) en Perú y Movimento Nacional do Meninhos e Meninhas de Rua (MNMMr) en Brasil. Respecto a la Convención Iberoamericana de los Derechos de los Jóvenes (CIDJ), firmada por el Estado mexicano en el año de 2005, también está precedida de la Declaración sobre el Fomento entre la Juventud de los Ideales de Paz, Respeto Mutuo y Comprensión entre los Pueblos (1965) en la ONU; el Año Internacional de la Juventud (1985); el Día Internacional de la Juventud (1999), el Programa de Acción Mundial hacia los Jóvenes (1995), y las Conferencias Intergubernamentales desde 1988. Es importante destacar que en México, de acuerdo con el artículo 133 constitucional, los tratados internacionales "se ubican jerárquicamente debajo de la Constitución Federal y por encima de las leyes generales, federales y locales, en la medida en que el Estado mexicano al subscribirlos, de conformidad con lo dispuesto en la Convención de Viena sobre el Derecho de los Tratados entre los Estados y Organizaciones Internacionales o entre Organizaciones Internacionales y, además, atendiendo al principio fundamental de derecho internacional consuetudinario pacta suntservanda, contrae deliberadamente obligaciones frente a la comunidad internacional” (REDIM, 2009: 11)

${ }^{2}$ La CDN define "niño" como ser humano menor a dieciocho años de edad, categoría jurídica que se ha armonizado con la Ley General de los Derechos de Niñas, Niños y Adolescentes (2014) y la Ley de los Derechos de Niñas, Niños y Adolescentes del Estado de Veracruz (2018). La UNICEF-México, por su parte, define a niñas y niños con base en rangos de edad: primera infancia (0 a 5 años), niñez o edad escolar (6 a 11 años) y adolescencia (12 a 17 años): http://www.unicef.org/mexico/spanish/. Respecto a las juventudes, en el Año Internacional de la Juventud (1985), se clasificó a la persona joven entre los 15 y 24 años, de igual forma que en la CIDJ. 
interés adulto, los mecanismos de integración social y las formas de socialización para la producción de subjetividades. Las relaciones intergeneracionales son relaciones de fuerza asimétricas (Foucault, 2008), mediadas por las transformaciones estructurales en los modos de regulación social producto de los modelos de acumulación de capital.

Desde este ángulo de análisis, las representaciones sociales y posiciones diferenciadas de la niñez y la juventud en las sociedades modernas implican una comprensión multicapilar, que conecte la reconstrucción histórica de la aparición de la infancia y juventud como objeto de socialización, protección y responsabilidad moral y las mutaciones del régimen de gubernamentalidad múltiple (Foucault, 1996), desplegado en las instituciones estatales, familiares, religiosas y privadas.

El historiador francés Philippe Ariés (1987) ha denominado el "descubrimiento de la infancia", ${ }^{3}$ como un acontecimiento moderno, que giró en torno a la separación de la infancia del mundo adulto, a partir de un proceso de moralización, hacia el cuidado y vigilancia de los niños, por medio de su internamiento y disciplinamiento en la escuela y la familia. Es evidente que la historia de la infancia y la juventud no puede entenderse como un continuum espacio-temporal sin contradicciones ni discontinuidades. Sin embargo, el aporte del autor permite situar la discusión en la correlación entre la emergencia de la noción de sujeto, sus posibilidades de emancipación y la expansión de la crianza y educación, como objeto de intervención del Estado moderno, por medio de un proceso de institucionalización que se sostiene en el principio ideológico de la socialización en clave funcionalista. ${ }^{4}$

El espíritu de los tratados internacionales, que reconoce a los niños y adolescentes/jóvenes como sujetos de derechos, está en permanente tensión con las formas de protección estatales y familiares. El denominado cambio de paradigma del tránsito de objetos de tutela a sujetos titulares de derechos es problemático desde su conceptualización y en las prácticas de vida cotidiana de los jóvenes.

La pretensión universalista del régimen de derechos humanos define un tipo ideal de adolescencia y juventud moderna, que entra en contradicción con la pluralidad de

\footnotetext{
${ }^{3}$ Pese al reconocimiento de los aportes de Ariés, la historiografía contemporánea ha intentado alejarse de la visión lineal de la construcción histórica de la infancia (Sosenski y Jackson, 2012). A contrapelo, los estudios se concentran en la reconstrucción de la agencia infantil en contextos históricos diferenciados, el papel del Estado y la familia en la posición social de la infancia y; la relación dialéctica en las construcciones ideales de la infancia: la visión de los adultos y las experiencias vividas por los niños.

${ }^{4}$ Para Pilotti (2001), el principio ideológico de la socialización en las sociedades modernas tiene sus raíces filosóficas en el pensamiento liberal de pensadores como Jhon Locke, y en el estructural-funcionalismo, desde la influencia de la pedagogía moral de Durkheim y el proceso de socialización de Parsons (1959), como pautas de conducta, roles y mecanismos de recompensas y castigos para la integración social.
} 
experiencias juveniles en contextos de multiculturalidad y con los cambios en la mediación del Estado y los modelos de desarrollo.

Para Boaventura de Sousa (2010), la reconstrucción multicultural de los derechos humanos implica su reconceptualización; esto es, trascender su forma tradicional universalista de globalización desde arriba (localismo globalizado), por una forma de globalización contrahegemónica multicultural desde abajo. En términos de los derechos juveniles, implica un diálogo intercultural de las juventudes sobre los intersticios de los valores y su validez universal en escenarios y contextos multiculturales.

Pensar la pluralidad de experiencias juveniles es desmontar el discurso eurocéntrico tradicional de la socialización, como una forma de integración totalizante y unidireccional. A contrapelo, la socialización en movimiento, y como disputa, permite aproximarse a las tensiones y conflictos entre la heterogeneidad de grupos juveniles e intergeneracionales. Además de mostrarnos las formas en que los jóvenes crean sus propias formas de organización social a escala micro de su vida cotidiana que, de acuerdo con cada experiencia individual/colectiva juvenil, puede expresar mecanismos de reproducción de estructuras jerárquicas, de dominación y violencia, o romper estas formas de relación social. Desmitificar a los jóvenes, como víctimas o victimarios, es reconocer su capacidad de agencia para interiorizar el domino y la exclusión social y generar líneas de cruce o desbordamientos múltiples, aun en sus estrategias de reproducción social.

Desde el marco legal de los derechos infantiles y juveniles, es visible que existen avances exiguos y limitados respecto a la autonomía de los adolescentes y jóvenes. Los instrumentos fundacionales de derechos, como la Declaración de Ginebra sobre los Derechos del Niño (1924), mantenía su concepción de derechos, desde una orientación hacia la protección, la asistencia, la escolarización y la educación familiar. De igual forma, la Declaración sobre el Fomento entre la Juventud de los Ideales de Paz, Respeto Mutuo y Comprensión entre los Pueblos (1965) ponía énfasis en los principios diferenciados de educación para los jóvenes.

En la actualidad, se cuenta con dos instrumentos internacionales de derechos de las infancias y juventudes (CDN y CIDJ), que incorporan en sus contenidos y horizontes de aplicabilidad, la participación infantil y juvenil como eje para la titularidad de derechos.

La CDN combina derechos civiles, políticos, culturales y económicos para las niñas, niños y adolescentes. Se estructura en cuatro principios generales: el interés superior del niño (Art. 3), el principio de no discriminación (Art. 2), el derecho del niño 
a la vida, supervivencia y desarrollo (Art. 6) y el derecho a la participación (Art. 12) Como instrumento jurídico vinculante, establece las obligaciones de los Estados firmantes y asigna responsabilidades a la familia, la sociedad civil y la cooperación internacional en el cuidado y protección infantil. Su esencia es definir la condición jurídica de la niñez/adolescencia y establecer los mecanismos internacionales de monitoreo por parte del Comité de los Derechos del Niño (expertos internacionales en el tema), con la presentación de informes periódicos a cada país sobre los avances en la materia.

El aporte de la CDN con respecto a los instrumentos que le precedieron es un reconocimiento a la participación de los niños en los temas que les afectan y a su derecho a ser escuchados: libertad de opinión y a ser escuchados (Art. 12), libertad de expresión (Art. 13), libertad de pensamiento (Art. 14) y libertad de asociación (Art. 15).

La participación infantil y adolescente desde la visión normativa, se concentra en la libre expresión y en ser tomados en cuenta con la mediación familiar y estatal. La perspectiva del sujeto, desde el nivel de desarrollo, restringe la independencia jurídica con base en el principio de autonomía progresiva, que se define en la correlación entre el nivel de desarrollo de las infancias y el ejercicio de sus derechos de manera autónoma. Una especie de evolución progresiva de sus facultades para ampliar los márgenes de su autonomía con respecto al ejercicio de sus derechos. De manera puntual, la socióloga Lourdes Gaitán (2006) lo explica:

El grupo de derechos relativos a la participación de los niños en la sociedad, siendo escuchados, especialmente en los temas que les afectan, es el más novedoso, pero, a la vez, el más restringido y el menos desarrollado en la práctica. Incluidos bajo este epígrafe estarían los artículos que hacen referencia al derecho a la libertad de expresión, de pensamiento y de conciencia (con la guía de los padres), a que el niño debe ser escuchado en todo procedimiento legal o administrativo que le afecte (pero no puede reclamar sus derechos jurídicos o administrativos si no es por mediación de sus padres o representantes), a la libertad de asociación y de celebrar reuniones pacíficas (aunque nada se menciona respecto al desarrollo de actividades políticas, de elegir a sus representantes o de ser elegido). El trabajo, que es también una forma de participación en la vida social, no está reconocido para los niños desde el lado de libertades, sino desde el de la protección... La protección ocupa la parte más

\footnotetext{
${ }^{5}$ La CDN establece los derechos de la niñez y adolescencia por grupos: a) Derechos de Protección: garantizan a los niños la protección contra el maltrato, la explotación económica y sexual y la discriminación por motivos de raza, sexo, religión o condición de edad; b) Derechos de Provisión: se refieren al derecho de todos los niños a gozar de un desarrollo óptimo y de bienestar, a la educación escolar básica, a la asistencia médica y a condiciones de vida dignas como seres humanos; c) Derechos de Participación: se relacionan con el derecho a la libre información y a la expresión de su opinión, a la participación en las decisiones relativas a su bienestar y a reunirse pacíficamente y construir sus propias organizaciones; d) Derechos de Prevención: están relacionados con el derecho a detectar de forma temprana situaciones que pongan en riesgo el pleno disfrute de los derechos de los niños y niñas (Alfageme, Cantos y Martínez, 2003, p. 31).
} 
extensa del articulado y, su mero enunciado supone un doloroso recorrido por todas las situaciones que amenazan la vida de los niños. (p. 71).

La visión institucional/normativa de la participación de la CDN trastoca también a los jóvenes/adolescentes, con base en la clasificación por edad en la definición de juventud (15 a 24 años). Sin embargo, los mayores de 18 años (jóvenes/adultos) son sujetos de independencia jurídica; por lo tanto, desaparece la noción de protección integral por parte del Estado. La CIDJ ${ }^{6}$ es un instrumento internacional de derechos de la juventud, firmado por el Estado mexicano que reconoce a los jóvenes "como sujetos de derechos, actores estratégicos del desarrollo y personas capaces de ejercer responsablemente los derechos y libertades” (p. 10). En razón de su condición psicosocial, de factores físicos y de identidad, se amplía la noción de participación a la de ciudadanía integral, que se traduce en la participación política y social en los diversos ámbitos de interacción y organización juvenil (Arts. 18, 21).

El reconocimiento de los jóvenes/adolescentes y los jóvenes/adultos, como sujetos de derechos, es resultado de un proceso de cambio en su posición social en las sociedades modernas. Ello ha implicado ajustes en la normatividad e instrumentos legales que les confieren la titularidad y ejercicio de sus derechos, la cual, en la práctica, es experimentada por los jóvenes de manera contradictoria y en disputa en las relaciones de fuerza intergeneracionales.

Los instrumentos internacionales de derechos de la niñez, adolescencia y juventud han influido en la creación de instituciones estatales y privadas en México; la armonización de marcos legislativos federales y estatales; un corpus de mecanismos e instrumentos para la promoción y defensa de derechos y la incorporación del discurso de derechos humanos en las narrativas gubernamentales.

Desde una mirada sociológica, es pertinente preguntarnos ¿qué tipo de subjetividades produce el régimen de derechos humanos especiales en un modelo de acumulación posfordista/neoliberal? y ¿cuáles son las posibilidades de la política

\footnotetext{
${ }^{6}$ La CIDJ se basa en los principios de no-discriminación, el derecho a la paz, la igualdad de género y el protagonismo de la familia. Agrupa los derechos humanos de los jóvenes en derechos civiles y políticos, derechos económicos, sociales y culturales. Los antecedentes de la Convención, se pueden rastrear en las Conferencias Iberoamericanas de Ministros de la Juventud, que preceden los trabajos de la Organización Iberoamericana de la Juventud (1992), como un organismo internacional público interinstitucional, que articula la cooperación en materia de juventud en interfaces con los gobiernos, la sociedad civil y la comunidad global. Se conforma por los organismos oficiales de la juventud de veintiún países iberoamericanos, entre ellos México. Entre sus líneas de acción, se orientan a generar "opinión pública en torno a temáticas relevantes para Iberoamérica y el mundo, resaltando las perspectivas de las personas jóvenes. Asimismo, desarrolla programas, proyectos e iniciativas orientados a fortalecer las capacidades de las y los jóvenes, a generar conocimiento de vanguardia e incidir en el entorno para que las temáticas de juventud estén en el centro de las agendas": https://oij.org/.
} 
multicultural contrahegemónica de derechos juveniles ante las mediaciones estatales y legislativas de la participación juvenil?. Intentaremos dilucidar tales preguntas en el siguiente apartado.

PARTICIPACIÓN SOCIAL Y POLÍTICA DE LOS JÓVENES: MARCOS LEGISLATIVOS Y POLÍTICAS PÚBLICAS EN MÉXICO

Pensar en la gubernamentalidad de los jóvenes es recorrer un entramado institucional, legal y de políticas hacia las juventudes en México. Para nuestros fines, realizamos un recorte en los contenidos de participación social y política, en leyes y políticas públicas, como formas de integración, mediación e intervención estatal.

Delinear el contorno de los derechos juveniles y su participación implica situar el análisis en el régimen político y los modos de regulación social hacia los jóvenes en la trama neoliberal. De otra forma, se corre el riesgo de encapsular la gramática de la juventud y sus derechos, como un jardín de buenas conciencias y de imperativos morales, nublando las contradicciones y la tensión dialéctica entre las formas de ordenamiento social y la emancipación juvenil.

Parafraseando a Pilotti (2001), es pertinente comprender el "contexto del texto" de los derechos juveniles de participación, para orientar sus posibilidades y alternativas multiculturales, como grietas y sublevaciones de organización y acción de los jóvenes. Los derechos de participación de los jóvenes están contenidos por un modo de gubernamentalidad neoliberal/globalizada (Fraser, 2003), que responde al modelo de acumulación de capital posfordista o mercadista. ${ }^{7}$

Las implicaciones de este modelo en la vida social de las juventudes, tiene varias dimensiones de análisis. Por un lado, la flexibilización laboral que desmontó el derecho al pleno empleo y al salario, propio del modelo fordista/taylorista que sustentó el Estado de bienestar; ha generado la crisis del empleo, que se traduce en la precarización laboral,

\footnotetext{
${ }^{7}$ Para comprender el transito del régimen de acumulación fordista al posfordista, el regulacionista (1994) Lipietz pone énfasis en los factores de crisis del modelo fordista, expresados en "el fin de la reducción de la parte exportada de la producción, la renovada importancia de las exportaciones”(p. 6), además de un proceso de internacionalización entre países fordistas, orientado a una forma de unificación económica con regulación internacional y acuerdo nacional (salario, legislación laboral, Estado benefactor), que entraba en contradicción con las tendencias competitivas de los países, mientras la ampliación de la exportación medraba el mercado interno con la disminución de la tasa de productividad, pese al aumento de la mecanización como paradigma tecnológico. Como soluciones a la crisis del fordismo, Lipietz identifica el paso de la rigidez de este modelo a un esquema flexible que rompa el acuerdo capital-trabajo (contratación colectiva), mediante una política monetarista, el desmantelamiento del Estado benefactor y la incorporación de la robotización a la organización del trabajo bajo el esquema taylorismo-mecanización-robotización.
} 
formas de contratación flexibles/ sin seguridad social, desempleo juvenil ${ }^{8}$ y el empleo informal.

Otra dimensión analítica es la dispersión de la gubernamentalidad globalizada, lo que significa la desnacionalización/trasnacionalización de las formas de regulación y ordenamiento social, que en el modelo fordista era una forma de ordenamiento disciplinario concentrado en el Estado-nación. El reflejo de este cambio se expresa en la extensión de la estatalización, a partir de una gubernamentalidad de múltiples capas y niveles mediados por el mercado. En este sentido, las agencias nacionales (seguridad, cobertura económica, bienestar social, derechos humanos) se articulan y descentralizan a escala internacional y transnacional con una pluralidad de actores, lo que representa una estructura disgregada.

En el caso del campo juvenil en México, esto es evidente en las formas de cooperación que se establecen entre el Instituto Nacional de la Juventud y los institutos estatales/municipales; el Sistema de Protección Integral de Niñas, Niños y Adolescentes (SIPINNA), los organismos de cooperación internacional y agencias del Sistema de Naciones Unidas (Fondo de las Naciones Unidas para la Infancia [UNICEF], Organización Internacional del Trabajo [OIT], Organización Iberoamericana de la Juventud, Agencia de la ONU para Refugiados [ACNUR], ONU-Habitat, entre otras); las organizaciones multinacionales especializadas en finanzas y asistencia financiera (Banco Mundial '[BM], FMI, Unión Europea [UE]); las organizaciones internacionales de derechos humanos (Save the Children, World Vision); las organizaciones nacionales en derechos de la adolescencia y la juventud, y las organizaciones de representación juvenil.

Respecto a la dimensión de desestructuración de lo social —entendido como formas de relación e interacciones sociales_-, la producción de la subjetividad juvenil está interiorizada por una sociabilidad individual (Chacón, 2017), que se despliega en la competitividad de las reglas del mercado, el individualismo absolutista, la competencia, la eficiencia, la construcción del agente activo responsable y la relación con el otro, desde la noción de amenaza o riesgo múltiple como componente en la interacción social.

\footnotetext{
${ }^{8}$ De acuerdo con el Informe Tendencias Mundiales del Empleo Juvenil (2015) de la OIT, en 2014 había, a nivel mundial, 73,3 millones de jóvenes desempleados, que representan el 36,7\% de los desempleados en el mundo. En México, el Instituto Nacional de Estadística, Geografía e informática (INEGI), de acuerdo con la Encuesta Nacional de Ocupación y Empleo (ENOE), 2018, indicó que la tasa de desocupación de la población de 15 a 29 años es de 5.8\%, superior a la nacional, de 3.2\%; la de 15 años y más es de $3.2 \%$ a nivel nacional, es decir, de 1.7 millones de personas, de las cuales, $60.8 \%$ son hombres y $39.2 \%$ son mujeres. 15 millones de personas jóvenes ocupadas de 15 a 29 años, 59.5\% labora en el sector informal. La Encuesta Nacional de Juventud (2010) expone que en el Estado de Veracruz se concentra el 6.6\% de los jóvenes en México, de los cuales el 21.7\% , de entre 14 y 29 años, no estudia ni trabaja, ocupando el quinto lugar a nivel nacional
} 
Es evidente que los cambios en la mediación del modelo neoliberal se imprimen a nivel del régimen político y sus políticas públicas y que, al mismo tiempo, trastocan las formas de reproducción social de la población juvenil. Los jóvenes, en México, han experimentado la crisis del régimen político, que ha implicado la integración subordinada en programas públicos de corte adultocéntrico y acciones de criminalización y represión para sectores juveniles disidentes o en condiciones de exclusión social.

La política contemporánea hacia los jóvenes en México es fruto del proyecto neoliberal que se instauró en el viejo régimen autoritario, propiciado por las crisis financieras de los años ochenta y noventa, las reformas del Estado, la privatización de los servicios de bienestar social y la política económica global.

A pesar de los procesos de alternancia de gobierno en el nivel federal y estatal, la génesis neoliberal ha mantenido su influencia en la política social hacia los jóvenes, delineada por la focalización en programas de combate a la pobreza, el asistencialismo o la subsidiariedad y los nuevos esquemas de participación ciudadana y gobernanza institucional.

En la estructura institucional, a finales de los años noventa, se creó el Instituto Mexicano de la Juventud (IMJ) ${ }^{9}$, un organismo público descentralizado de la Administración Pública Federal, con la finalidad de asegurar a la juventud mexicana su pleno desarrollo integral y bienestar en condiciones de equidad y no discriminación, a partir del diseño e implementación de la política nacional de juventud. En 2016, surge SIPINNA, como órgano de articulación y concurrencia de la política de protección integral de derechos hacia la niñez y adolescencia. Ambas estructuras, impulsadas por mandato de ley, ${ }^{10}$ resultado de las luchas por el reconocimiento de los adolescentes y jóvenes como sujetos de derechos.

\footnotetext{
${ }^{9}$ Como antecedentes a la creación del IMJ, el Estado mexicano tuvo el Instituto Nacional de la Juventud Mexicana (INJM 1950), fundado durante la presidencia de Miguel Alemán Valdez con el objeto de capacitar a los jóvenes para el sector industrial del mercado laboral. Posteriormente, en la administración de José López Portillo se modificó el INJM por la creación del Consejo Nacional de Recursos para la Atención de la Juventud (CREA-1977). En el periodo de Carlos Salinas de Gortari, el CREA desapareció y, en su lugar, se instaló la Dirección General de Atención a la Juventud en la Comisión Nacional del Deporte; más adelante, con Ernesto Zedillo Ponce de León se creó “Causa Joven”, instancia encargada de promover medidas legislativas para la juventud.

${ }^{10}$ El SIPINNA fue creado con base en la Ley General de los Derechos de Niñas, Niños y Adolescentes (2014), que es resultado de un proceso de armonización legislativa de treinta años en el país, que transitó por reformas legislativas a la Constitución política mexicana ( $4^{\circ}-1999,1^{\circ}$ y $\left.2^{\circ}-2001,3^{\circ}-2002,73^{\circ}-2011\right)$; la aprobación de la Ley de Protección de los Derechos de las Niñas, Niños y Adolescentes de carácter federal (2000), y la adecuación de la legislación estatal, que en Veracruz comenzó con la Ley de Protección de los Derechos de Niñas, Niños y Adolescentes para el Estado de Veracruz (2008) y la Ley de los Derechos de Niñas, Niños y Adolescentes del Estado de Veracruz (2015). La creación del IMJ se fundamenta en la Ley del Instituto Mexicano de la Juventud (1998). Actualmente se trabaja en la elaboración de una Ley General de Juventudes por parte de la Comisión de Juventud y Diversidad Sexual de la Cámara de Diputados; a nivel estatal se cuenta con la Ley de Desarrollo Integral de la Juventud (2005).
} 
SIPINNA busca superar la tradición asistencialista del Sistema para el Desarrollo Integral de la Familia (DIF) en la atención a la niñez y adolescencia, con un modelo basado en la integralidad de la política de Estado, mientras el Instituto de la Juventud se orienta a ampliar los mecanismos de integración social y construcción de capacidades juveniles en los ámbitos de salud, educación, empleo y participación política y social. A pesar de sus diferencias en composición y forma, el hilo conductor estructural de su configuración institucional está en su modelo de gobernanza, que implica la creación de espacios y mecanismos de participación de la sociedad civil y fundamentalmente de los adolescentes y jóvenes; ejemplo de ello, su estructura política.

El IMJ cuenta con una junta directiva, integrada por diecisiete miembros, con una composición asimétrica entre los representantes de la administración pública federal/estatal, dos representantes de universidades y dos jóvenes del Consejo de Seguimiento de Proyectos y Programas. SIPINNA, por su parte, cuenta con once representantes de la administración pública federal/estatal, tres de organismos públicos, ocho de sociedad civil y ocho niñas, niños y adolescentes, invitados especiales con voz, pero sin voto.

El Instituto cuenta con el Consejo Ciudadano de Seguimiento de Proyectos y Programas, como órgano que monitorea el cumplimiento de los programas hacia la juventud; está integrado por veinte jóvenes mayores de edad. En el SIPINNA, hay una serie de mecanismos participativos para la sociedad civil, como el Consejo Consultivo y las comisiones de trabajo especializadas, que no incluyen a adolescentes.

Otro elemento de la configuración institucional es la concurrencia entre los diferentes niveles de gobierno. Tanto el Instituto como el Sistema cuentan con instancias similares a nivel estatal y municipal que responden a las líneas y ejes de trabajo de la administración central, respetando algunos márgenes de autonomía en torno a su estructura política, administrativa y operativa. ${ }^{11}$

La participación adolescente y juvenil es parte de la reingeniería institucional del gobierno, que se sostiene en el discurso democrático y la participación institucionalizada. Sus alcances para la toma de decisiones estructurales aún son cuestionables con diversos matices. Es manifiesta la integración de adolescentes y jóvenes en espacios y mecanismos para el diálogo, seguimiento y generación de propuestas para la política de Estado. Sin embargo, esta forma de participación estatalizada reproduce las representaciones sociales

${ }^{11}$ En el Estado de Veracruz, hubo una modificación durante la Administración de Miguel Ángel Yunes Linares (20162018), que transformó la Subsecretaria de la Juventud, perteneciente a la Secretaria de Gobierno en el Instituto Veracruzano de la Juventud, como órgano desconcentrado de la Oficina del Gobernador. 
adulto-céntricas que se delinean en sus convocatorias, formatos y contenidos, como se ejemplifica en las acciones: "Diputado infantil por un día”, "Delegados Juveniles", “Consejeros Juveniles”, "Parlamento Juvenil”, "Premio Nacional de la Juventud”, entre otros.

Estos esquemas de participación política juvenil filtran y segmentan la pluralidad de experiencias juveniles, porque se fundamentan en perfiles y capacidades de tipos ideales de juventud o responden a intereses políticos de incorporación de cuadros afines al interés del partido en turno. En contextos de acumulación de desigualdades y encadenamientos de violencias, las juventudes quedan excluidas de los criterios y requerimientos de las convocatorias. Además, la crisis del régimen político ha limitado las expectativas y motivaciones de construcción de ciudadanía juvenil y de cambio en la incidencia y participación en la política de Estado; de hecho, los horizontes de las juventudes marginadas y excluidas están en estrategias de supervivencia, como la reconstrucción de su capacidad de agencia en entornos complejos y adversos. Así pues, la participación política juvenil en la estructura estatal es fragmentaria y de acceso a ciertos sectores juveniles.

Existen otras formas de participación, establecidas en los tratados internacionales y marcos legislativos que se diseminan en las políticas públicas. Tanto la CIDJ como la CDN reconocen los derechos de los adolescentes y jóvenes a la libertad de expresión, de asociación y reunión (Arts.18, 71-75,); la particularidad en este instrumento internacional juvenil es que incluye el derecho a "disponer de foros juveniles y a crear organizaciones y asociaciones [...] les posibiliten la obtención de recursos concursables para el financiamiento de sus actividades, proyectos y programas" (p. 22).

Las directrices internacionales se incorporan en la legislación y en acciones concretas de las políticas del Estado mexicano. El Programa Nacional de Juventud es el instrumento rector que establece la definición e instrumentación de la política nacional hacia los jóvenes, recuperada e implementada por los planes estatales y programas municipales. De igual forma, existe el Programa Nacional de Protección Integral de Niñas, Niños y Adolescentes (PRONNAPINA), que establece las estrategias y líneas de trabajo para la protección integral a los adolescentes, se articula y coordina con los programas de nivel estatal y municipal.

En Veracruz, se mantiene la ausencia de un programa estatal de protección integral a niñas, niños y adolescentes. En materia de participación, se cuenta con un documento de carácter federal, creado por el SIPINNA, denominado "Lineamientos para la Participación de Niñas, Niños y Adolescentes en México" (2016), cuyo objeto es 
"garantizar la participación permanente, sistemática y activa de Niñas, Niños y Adolescentes en el diseño y evaluación de las políticas públicas, especialmente aquellas que tengan un impacto directo para su vida y desarrollo óptimo, así como promover su participación en los ámbitos familiar, escolar, comunitario, social o cualquier otro en el que se desarrollen" (p. 3).

El aporte de dicho documento es establecer los estándares mínimos de la participación y el proceso participativo, mediante la orientación de las condiciones básicas o principios participativos (libertad de opinión, autonomía progresiva, inclusión, igualdad y no discriminación), y las características que debe asumir un proceso participativo (voluntario, informado, temas de interés, mecanismos de devolución).

Es pertinente apuntar que los procesos participativos institucionalizados se fundamentan en la noción de autonomía progresiva y en la concepción de la participación gradual $^{12}$ (Hart, 1993), un paradigma evolutivo y lineal de acumulación de facultades, hacia la construcción de ciudadanía formal.

La participación infantil es incorporada en el discurso democrático de los actores gubernamentales. En la práctica, existen esfuerzos de consultas infantiles, lineamientos y foros para la integración de la niñez y adolescencia en el SIPINNA, que distan de ser considerados expresiones de una participación protagónica. En cuanto a la política hacia los jóvenes, el Plan Nacional de Juventud ha sido el eje rector de las políticas estatales y municipales, resultado de un modelo de política de Estado centralizado, que eclipsa las texturas regionales y realidades locales.

En contraste con la política infantil, el espectro de acciones de participación social y política de los jóvenes es más amplio. Como hemos referido, la participación política se expresa en espacios de interacción y diálogo con respecto a las condiciones de vida juvenil y políticas públicas a partir de mecanismos políticos tradicionales de representación: consejos, parlamentos, foros y consultas.

Por otro lado, la participación social y comunitaria se ha centrado en programas de radio y televisión (Programa "Poder Joven"); capacitación y espacios de encuentro para el liderazgo, capital social, asociacionismo/organización y emprendimiento

${ }^{12}$ Para Roger Hart, existen ocho niveles y gradualidades de participación: Nivel 1: Manipulación y engaño; Nivel 2: Decoración y Escaparate; Nivel 3: Participación simbólica o apariencia; Nivel 4: Asignados pero informados; Nivel 5: Consultados e informados; Nivel 6: Iniciados por los adultos, compartidas con los niños; Nivel 7: Iniciados y dirigidos por los niños, y Nivel 8: Iniciados y dirigidos por los niños, decisiones compartidas con los adultos. 
juvenil, ${ }^{13}$ así como en el apoyo a proyectos sociales de colectivos juveniles, a partir del fondo PROJUVENTUDES y de Proyectos Locales Juveniles.

En resumen, la política hacia la adolescencia y juventud en México está contenida por un modo de gubernamentalidad globalizada, cuyos instrumentos de articulación y cooperación internacional han sido el Programa de Acción Mundial para los Jóvenes de las Naciones Unidas (1995), los Objetivos de Desarrollo del Milenio (2000) y los Objetivos de Desarrollo Sostenibles: jóvenes hacia el 2030.

Los nodos de concurrencia contituyen un entramado complejo entre las agencias y directrices del Sistema de Naciones Unidas, las entidades gubernamentales y los programas de organismos de cooperación internacional. ${ }^{14}$ A nivel del Estado mexicano, la tradición centralista de la administración pública entra en tensión con la heterogeneidad de estructuras de los gobiernos estatales y municipales y con la diversidad de realidades y experiencias juveniles. La política juvenil es una zona gris de enfoques e imaginarios gubernamentales sobre los jóvenes y la orientación de las políticas, que oscilan entre la juventud como etapa preparatoria, como etapa problemática (criminalización y estigma social), la ciudadanía juvenil (sujetos de derechos) y el conglomerado de actores del desarrollo, a partir de su capital humano/social y la formación de capacidades (Krauskopf, 2003).

El agente activo responsable es el componente central de la participación política y social estatalizada hacia los jóvenes. La juventud, como protagonista del desarrollo, se nutre de un conjunto de programas y acciones orientados al emprendimiento social, el cual produce un modo de subjetividad juvenil, centrada en el liderazgo y la innovación social, que utiliza estrategias de mercado para alcanzar alternativas de solución a objetos sociales de interés.

Las formas gubernamentales de integración neoliberal producen un individuo juvenil obligado a mejorar sus condiciones de vida, mediante sus propias decisiones y el desarrollo de su capital humano. La participación política y social se subordina a la reproducción de un sujeto político juvenil, estructurado por mecanismos y prácticas políticas del régimen tradicional adulto-céntrico, con la intención de formar líderes

\footnotetext{
${ }^{13}$ De acuerdo con el Informe del Programa Nacional de Juventud (2014-2018), las acciones de participación dirigidas hacia los jóvenes fueron: Impulso México (IMJUVE-SEDESOL), Concurso Juvenil de Debate Político (INE-IMJUVE), Cultura de Protección Civil para Organizaciones de la Sociedad Civil (SEGOB), RUMBO JOVEN Cumbre de Jóvenes, Segundo Campamento Nacional de Jóvenes del Movimiento Sin Odio "Narrativas de Odio, juventudes y transformación social" (CONAPRED, OEA, OEI, COPRED), Encuentro Nacional "Ritmo Joven: lazos de inclusión”, Encuentro Nacional “PODER JOVEN”, Tutores Comunitarios de Verano (SEP, CONAFE, PROSPERA), JOVEN, A.C, Joven- Es Compromiso: Proyectos Sociales Comunitarios.

${ }^{14}$ Un ejemplo son los programas de la Organización Iberoamericana de la Juventud (OIJ), que se replican en las entidades gubernamentales: Tarjeta Joven, Premios Juventud, Yo Puedo Emprender, Generación 2030-Programa de Liderazgo Político Joven.
} 
socializados en el híper-individualismo. El sujeto social es reducido a un asociacionismo formal e institucionalizado, que prioriza la racionalidad instrumental y tecnificación de colectividades, con base en la innovación y creatividad para la procuración de fondos y la gestión de problemas por modelos de mercado.

Las tensiones y paradojas de la política social hacia los jóvenes (Alvarado, Rodríguez y Vommaro, 2013) se expresan en las contradicciones de la participación juvenil; mientras se promueve el liderazgo juvenil en esquemas de representación artificiales, los movimientos juveniles, con agendas de cambio sistémico, quedan excluidos de la política juvenil. La producción de un sujeto juvenil homogéneo y neoliberalmente responsable desmonta la pluralidad de experiencias de los jóvenes y desactiva el pensamiento crítico.

El gobierno en turno tiene el desafío de invertir el centralismo de la política juvenil y transitar a una política multicultural de derechos juveniles. La apuesta por el programa de transferencias "Jóvenes Construyendo el Futuro" no puede ser un fin en sí mismo, debe acompañarse de procesos de integración diferenciados que recuperen las inquietudes y plataformas de la pluralidad de movimientos y culturas juveniles.

Los programas implementados, como la Consulta Nacional "República Joven”, los Encuentros Estatales por la Juventud, los Foros Regionales para la Incidencia en Políticas Públicas, las Brigadas Comunitarias de Norte a Sur, Territorio Joven, la Red Nacional de Radio y Televisión "Dilo Fuerte", entre otros, no deben reproducir las paradojas de la política juvenil, ${ }^{15}$ ni reducir la participación social y política institucionalizada a una narrativa neoliberal de liderazgo juvenil individualizado.

\section{CONSIDERACIONES FINALES}

A lo largo de este trabajo, se trazan las coordenadas de análisis sobre las formas estatales de participación social/política de los adolescentes y jóvenes, condensadas en la trama neoliberal en México. Apuntamos, en clave sociológica, que los procesos de integración juvenil se han trastocados por las nuevas formas de flexibilización laboral, la negación del

\footnotetext{
${ }^{15}$ En el texto de Alvarado, Rodríguez y Vommaro (2013): Políticas públicas de juventud e inclusión social en América Latina y el Caribe, se establecen las paradojas en el funcionamiento de las políticas públicas hacia los jóvenes: las políticas sectoriales y su enfoque de riesgo en tensión con el reconocimiento como sujetos de derecho/actores del desarrollo; la contradicción entre los documentos de políticas públicas de juventud que priorizan la inserción laboral y la ciudadanía, con los presupuestos públicos de integración a la educación y uso del tiempo libre; la creación de espacios específicos para la juventud sin una perspectiva generacional; la escasa incidencia efectiva de los pequeños proyectos sectoriales y las leyes generales de juventud, e Institutos de la Juventud caracterizados por la contratación de "lideres" juveniles, más interesados en sus trayectorias políticas que en las políticas públicas para la juventud, entre otras.
} 
acceso al empleo, la acumulación y encadenamientos de violencias/desigualdades, las relaciones de poder asimétricas, intergeneracionales, y la producción de subjetividades juveniles desde el agente activo responsable del emprendimiento social.

La mediación estatal para el impulso de una ciudadanía juvenil reproduce los esquemas de la cultura política hegemónica, que se traduce en mecanismos institucionales de integración subordinada para algunos, y de exclusión sistemática para las juventudes precarizadas y excedentes del sistema. Problematizar el universalismo de los derechos humanos de los adolescentes y los jóvenes, la centralización de las políticas juveniles y los límites de la participación juvenil institucionalizada es abrir un horizonte para la discusión de las formas de participación protagónica no institucionales y los nuevos espacios de sociabilidad en la vida cotidiana de los adolescentes y los jóvenes.

Rastrear las prácticas de sociabilidad de la heterogeneidad juvenil es adentrarse en una pluralidad de experiencias situadas en los ámbitos familiares, vecinales, comunitarios y en los espacios de las nuevas tecnologías de la información. La apropiación simbólica y los significados de la participación juvenil en contextos de violencia, vulnerabilidad y exclusión social se reconfiguran y actualizan en las experiencias de supervivencia, que condensan una identidad multicultural de formas de organización, interacciones y generación de estrategias de resistencia ante el devenir histórico de las formas de dominación, segregación urbana y exclusión social.

La socialización política de las juventudes implica deconstruir la noción de "lo político juvenil” como campo homogéneo, evolutivo y unidireccional, que se presenta encapsulado ante las tensiones y relaciones de poder generacionales e intergeneracionales y las dimensiones estructurales y sistémicas que trastocan la vida juvenil. Se trata de reconocer las contradicciones inherentes a la socialización y la multiplicidad de estrategias de participación juvenil, a nivel capilar, en escenarios múltiples.

\section{REFERENCIAS}

Alfageme, E., Cantos, R., Martínez, M. (2003). De la participación al protagonismo infantil. Madrid: Plataforma de Organizaciones de Infancia.

Alvarado, Rodríguez y Vommaro (2013). Políticas públicas de juventud e inclusión social en América Latina y el Caribe. Buenos Aires, Argentina: UNESCO, ClACSO.

ARIÉS, P. (1987). El niño y la vida familiar en el Antiguo Régimen. Madrid: Taurus. 
Auyero, J., y Berti, M. F. (2013). La violencia en los márgenes. Buenos Aires, Argentina: Katz.

Bourdieu, P. (1990). Sociología y Cultura. México: Grijalbo,

Chacón, J. (2017). Regulación neoliberal. Magistra, 14.

Convención Iberoamericana de los Derechos de los Jóvenes de la Organización Iberoamericana de la Juventud (2005).

Convención Sobre los Derechos del Niño (1989). Asamblea de las Naciones Unidas.

De Sousa Santos, B. (2010). Descolonizar el saber, reinventar el poder. Uruguay: TRILCE.

DUBET, FRANÇOIS (2007). La experiencia sociológica. Madrid: Gedisa.

Encuesta Nacional de Juventud (2010). México: Instituto Mexicano de la Juventud (IMJ).

Encuesta Nacional de Ocupación y Empleo (2018). México: Instituto Nacional de Estadística, Geografía e Informática (INEGI).

Foucault, M. (2008). Historia de la Sexualidad: La Voluntad de Saber. Buenos Aires, Argentina. Siglo XXI Editores.

FRASER, N. (Enero-abril, 2003). ¿De la disciplina hacia la flexibilización? Releyendo a Foucault bajo la sombra de la globalización. Revista Mexicana de Ciencias Políticas y Sociales, XLVI(187), pp. 15-33.

GaitÁn, L. (2006). La nueva sociología de la infancia. Aportes de una mirada distinta. Política y Sociedad,1(43), pp. 9-26.

Guerra Ramírez, M. I. (2009). Trayectorias formativas y laborales de jóvenes de sectores populares: Un abordaje biográfico. México: ANUIES.

HART, R. (1993). La participación de los niños: de la participación simbólica a la participación autentica. Bogotá: UNICEF.

Informe de Avances y Resultados del Programa Nacional de Juventud (2014-2018). México: Instituto Mexicano de la Juventud.

Informe Invertir para Transformar (2014). Organización Iberoamericana de Juventud, Comisión Económica para América Latina y el Caribe (CEPAL), Instituto Mexicano de la Juventud, Organización Internacional del Trabajo (OIT), Centro Latinoamericano sobre Juventud (CELAJU), Banco de Desarrollo en América (CAF) Latina, Agencia Española de Cooperación Internacional para el Desarrollo (AECID).

Informe Tendencias Mundiales del Empleo Juvenil (2015). OIT.

KRAUSKOPF, D. (2003). La construcción de políticas de juventud en Centroamérica. En:

Políticas públicas de juventud en América Latina: políticas nacionales. Viña del Mar: CIDPA.

LAHIRE, B. (2004). El hombre plural: Los resortes de la acción, Bellaterra, Catalunya. 
Ley de los Derechos de Niñas, Niños y Adolescentes del Estado de Veracruz (2018). Xalapa: Congreso del Estado de Veracruz.

Ley de Desarrollo Integral de la Juventud (2005). Xalapa: Congreso del Estado de Veracruz.

Ley General de los Derechos de Niñas, Niños y Adolescentes (2014). México: Congreso de la Unión, Senado de la República.

Ley del Instituto Mexicano de la Juventud (1998). México: Congreso de la Unión.

Ley de Protección de los Derechos de las Niñas, Niños y Adolescentes (2000). México: Congreso de la Unión.

Ley de Protección de los Derechos de Niñas, Niños y Adolescentes para el Estado de Veracruz (2008). Xalapa: Congreso del Estado de Veracruz.

Liebel, M. (2006). Experto en Políticas Sociales de Infancia. En: Entre protección y emancipación. Derechos de la infancia y políticas sociales, 1. Madrid: UCM-Facultad de Ciencias Políticas y Sociología.

Lineamientos para la Participación de Niñas, Niños y Adolescentes en México (2016). México: Sistema Nacional de Protección Integral de Niñas, Niños y Adolescentes (SIPINNA).

LiPIETZ, A. (1994). El posfordismo y sus espacios: las relaciones capital-trabajo en el mundo, Buenos Aires, Argentina: PIETTE.

MisSE, M. (julio-diciembre, 2010). La acumulación social de la violencia en Río de Janeiro y en Brasil: algunas reflexiones. Co-herencia, 7(13). pp. 19-40.

Objetivos de Desarrollo Sostenible, Innovación para la transformación: Jóvenes hacia el 2030 (2018). Instituto Mexicano de la Juventud, Fondo de Población de las Naciones Unidas, México.

PilotTi, F.(2001). Globalización y Convención sobre los Derechos del Niño: el contexto del texto. Santiago de Chile: ONU.

Programa Nacional de la Juventud (2019-2024). México: Instituto Mexicano de la Juventud. Programa Nacional de Protección Integral de Niñas, Niños y Adolescentes (2016-2018). México: Sistema Nacional de Protección Integral de Niñas, Niños y Adolescentes (SIPINNA).

SARAVÍ, G. (2015). Juventudes fragmentadas: socialización, clase y cultura en la construcción de la desigualdad. México: FLACSO, CIESAS.

SOSENSKI, S., Y JACKSON, E. (2012). Nuevas miradas a la historia de la infancia en América Latina: entre prácticas y representaciones. México: UNAM-Instituto de Investigaciones Históricas, México, Leyes e informes. 